\title{
Laboratory Test Reference Range Upper Limit Original Unit
}

National Cancer Institute

\section{Source}

National Cancer Institute. Laboratory Test Reference Range Upper Limit Original Unit. NCI Thesaurus. Code C83073.

The upper limit of the laboratory test reference range, as reported in original units. 